Chapter 2

\title{
RANS Numerical Simulation of Turbulent Particulate Pipe Flow for Fixed Reynolds Number
}

\author{
Alexander Kartushinsky, Ylo Rudi, Igor Shcheglov, \\ Sergei Tisler and Igor Krupenski
}

Additional information is available at the end of the chapter

http://dx.doi.org/10.5772/57216

\section{Introduction}

Particulate flows in pipes have numerous engineering applications ranging from pneumatic conveying systems to coal gasifiers and chemical reactor design and are one of the most thoroughly investigated subjects in the area of multiphase flows. These flows are very complex and influenced by various physical phenomena, such as particle-turbulence and particleparticle interactions, deposition, by gravitational and viscous drag forces, particle rotation, and lift force.

Numerous theoretical and experimental researches, e.g., [1-20], studied various aspects of the behavior of gas and solid particles in particulate pipe flows.

The present study focuses on the effect of variation of the pipe diameter for a constant Reynolds number applied to vertical particulate turbulent pipe flows. The numerical investigation discussed here examined in detail the effects of direct and indirect particle-turbulence interaction (no-coupling and coupling) and gravity for various flow mass loadings of 250, 500 and $700 \mu \mathrm{m}$ coal particles. Additionally, the viscous drag force and the Magnus and Saffman lift forces are also taken into account. The behavior of the particulate phase was under consideration, both for the fine particles being liable to the turbulent fluctuations of gas and the larger particles, which have the lesser response to the flow turbulence.

The presented numerical model makes use of the two-fluid model, e.g., [21-25], and the Reynolds-averaged Navier-Stokes (RANS) approach $[18,19]$ applied to gas and solid particles.

Within the frame of the two-fluid model, the gas and the particles are considered as two coexisting phases that span the entire flow domain $[18,19]$. Therefore, in order to describe the 
flow of the particulate phase within the two-fluid model, the presented model implements the RANS approach. This approach is the most general and frequently used in modeling, its closure equations have been verified by numerous experiments, and the boundary conditions are easy to determine. The given modeling employs the model [14], which is the most relevant to account for mechanisms of a turbulence modulation caused by particles, since it includes both the turbulence enhancement and its attenuation by particles. The inter-particle collisions is another mechanism accounting for capture properties of turbulent particulate pipe flows, which has been modeled, e.g., in [16]. These two models enables comprehensive mathematical simulation of the two-phase upward pipe flow.

The presented model allows covering 100 and more calibers of a pipe flow. This is the main advantage over the numerical models based, for example, on direct numerical simulation (DNS) codes, (e.g., [26]), that handle usually with a short pipe length up to 10-20 calibers with imposing the upper limit for the flow Reynolds number.

The utilized two-fluid model with adoption of original collisional closure model [16] together with the applied numerical method has been verified and validated in our previous researches $[18,19]$ by comparison of numerical results with the experimental data [6]. In the given study, the effect of variation of the pipe diameter (or transport velocity) at a constant Reynolds number is numerically investigated in the particulate turbulent flow. This is a step forward for analyzing the external effect, namely, the flow configuration rather the internal effect with variation of the parameters of the flow.

\section{Governing equations and numerical method}

The sketch of the computational flow domain is shown in Fig. 1, where $\bar{u}$ is the gas average velocity, $F_{G}$ is gravity, $F_{D}$ is the aerodynamic drag force, $F_{L R}$ is the lift force that arises from particle rotation (the Magnus lift force), $\omega_{s}$ is the angular velocity of a particle.

It is assumed that the particulate phase is polydispersed and composed of several known mass fractions. These fractions can be of single material density and characterized by equivalent particle diameter of the fraction $\delta$. According to [16], in the given formulation of the governing equations that follows, three solid fractions are assumed to be present. It is assumed that the aerodynamic forces, such as the drag, lift forces and gravity, act on all the particulate fractions.

\subsection{Governing equations for the 2D RANS model}

The model is based on the time averaged Navier-Stokes equations (RANS method), without any simplifications, such as the boundary layer simplifications. The vertical pipe flows are 2D unless the study of rotating flows.

A short presentation of the governing equations written for the axisymmetric channel case is as follows: 


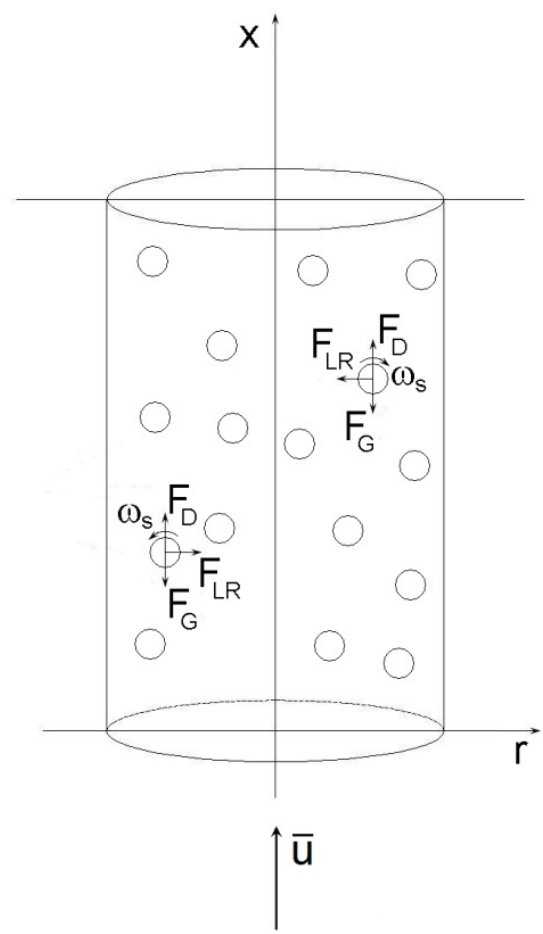

Figure 1. Upward turbulent particulate flow in a pipe.

continuity equation for the gas phase:

$$
\frac{\partial u}{\partial x}+\frac{\partial(r v)}{r \partial r}=0
$$

where $u$ and $v$ are the longitudinal and radial velocity components of the gas phase.

longitudinal linear momentum equation for the gas phase:

$$
\frac{\partial}{\partial x}\left(u^{2}-\tilde{v}_{t} \frac{\partial u}{\partial x}\right)+\frac{\partial}{r \partial r} r\left(u v-\tilde{v}_{t} \frac{\partial u}{\partial r}\right)=-\frac{\partial p}{\rho \partial x}+\frac{\partial}{\partial x} \tilde{v}_{t} \frac{\partial u}{\partial x}+\frac{\partial}{r \partial r} r \tilde{v}_{t} \frac{\partial v}{\partial x}-\alpha\left(\frac{u_{r}}{\tau^{\prime}}+C_{M^{\prime}} \Omega v_{r}\right)
$$

where $\tilde{v}_{t}=v_{t}+v$ is effective viscosity, which is the sum of turbulent and laminar viscosities, while $v_{t}$ is calculated following the Boussinesq eddy-viscosity concept; $p$ is pressure; $\alpha$ is the mass concentration of particles; $u_{r}=u-u_{s}$ and $v_{r}=v-v_{s}$ are the relative velocities of particles 
along the longitudinal and radial directions, respectively. Here $\tau^{\prime}=\tau / C^{\prime}{ }_{D}$ is the particle response time that specifies the drag, defined by the expression $C^{\prime}{ }_{D}=1+0.15 \operatorname{Re}_{s}^{0.687}$ for the nonStokesian regime [27]. The particle Reynolds number and Stokesian particle response time are defined as $\quad \operatorname{Re}_{s}=\delta\left|\vec{V}_{r}\right| / v=\delta \sqrt{u_{r}^{2}+v_{r}^{2}} / v \quad$ and $\tau=\rho_{p} \delta^{2} /(18 \rho v)$, respectively. $\Omega=\omega_{s}-0.5(\partial v / \partial x-\partial u / \partial r)$ is the angular velocity slip, with $\omega_{s}$ being the angular velocity of the given particle fraction. The coefficient of the Magnus lift force $C_{M}$ is calculated according to Crowe et al. (1998); $\rho$ and $\rho_{p}$ are the physical densities of air and the particle material, respectively.

radial linear momentum equation for the gas phase:

$$
\begin{aligned}
& \frac{\partial}{\partial x}\left(u v-\tilde{v}_{t} \frac{\partial v}{\partial x}\right)+\frac{\partial}{r \partial r} r\left(v^{2}-\tilde{v}_{t} \frac{\partial v}{\partial r}\right)=-\frac{\partial p}{\rho \partial r}+\frac{\partial}{\partial x} \tilde{v}_{t} \frac{\partial u}{\partial r}+ \\
& +\frac{\partial}{r \partial r} r \tilde{v}_{t} \frac{\partial v}{\partial r}-\frac{2 \tilde{v}_{t} v}{r^{2}}-\alpha\left(\frac{v_{r}}{\tau^{\prime}}-\left(C_{M} \Omega+F_{s}\right) u_{r}\right) .
\end{aligned}
$$

$F_{s}$ is the coefficient for the Saffman lift force, which is due to the local shear of the flow; it is given for finite values of the particle Reynolds numbers by the correction [28].

turbulence kinetic energy equation for the gas phase:

$$
\begin{aligned}
& \frac{\partial}{\partial x}\left(u k-\tilde{v}_{t} \frac{\partial k}{\partial x}\right)+\frac{\partial}{r \partial r} r\left(v k-\tilde{v}_{t} \frac{\partial k}{\partial r}\right)= \\
& =2 v_{t}\left\{\left(\frac{\partial u}{\partial x}\right)^{2}+\left(\frac{\partial r v}{r \partial r}\right)^{2}+\frac{1}{2}\left(\frac{\partial u}{\partial r}+\frac{\partial v}{\partial x}\right)^{2}\right\}+\frac{\alpha}{\tau}\left(u_{r}^{2}+v_{r}^{2}+k_{s}\right)-\varepsilon_{h}
\end{aligned}
$$

where $k$ and $k_{s}$ are the turbulence kinetic energy of the gas- and particulate phases, respectively. The hybrid dissipation rate $\varepsilon_{h}$ is calculated for the two-phase flow via hybrid turbulence length scale defined as harmonic average of the integral length scale of single-phase flow and interparticle spacing [14].

continuity equation for the particulate phase:

$$
\frac{\partial}{\partial x}\left(\alpha \tilde{u}_{s}\right)+\frac{\partial}{r \partial r} r\left(\alpha \tilde{v}_{s}\right)=0
$$

where $\tilde{u}_{s}$ and $\tilde{v}_{s}$ are the longitudinal and radial components of the drift particle velocity of the given fraction, given by expressions $\tilde{u}_{s}=u_{s}-\left(D_{t}+D_{c}^{x}\right) \mathrm{\partial} \ln \alpha / \partial x, \tilde{v}_{s}=v_{s}-\left(D_{t}+D_{c}^{r}\right) \mathrm{\partial} \ln \alpha / \partial r$. Here $D_{t}$ is the coefficient of turbulent diffusion of particles, which is calculated by the model 
[29]. The pseudoviscosity diffusion coefficients along $x$ and $r$ directions $D_{c}^{x, r}$ stem from the particle collisions [16].

momentum equation in the longitudinal direction for the particulate phase:

$$
\frac{\partial}{\partial x}\left(\alpha u_{s} \tilde{u}_{s}\right)+\frac{\partial}{r \partial r}\left(r \alpha u_{s} \tilde{s}_{s}\right)=-\frac{\partial}{\partial x}\left(\alpha \overline{u_{s}^{\prime 2}}\right)-\frac{\partial}{r \partial r}\left(r \alpha \overline{u_{s}^{\prime} v_{s}^{\prime}}\right)+\alpha\left[\frac{u_{r}}{\tau^{\prime}}+C_{M^{\prime}} \Omega v_{r}-g\left(1-\frac{\rho}{\rho_{p}}\right)\right]
$$

where $g$ is the gravitational acceleration.

momentum equation in the radial direction for the particulate phase:

$$
\frac{\partial}{\partial x}\left(\alpha v_{s} \tilde{u}_{s}\right)+\frac{\partial}{r \partial r}\left(r \alpha v_{s} \tilde{v}_{s}\right)=-\frac{\partial}{\partial x}\left(\alpha \overline{u_{s}^{\prime} v_{s}^{\prime}}\right)-\frac{\partial}{r \partial r}\left(r \alpha \overline{v_{s}^{\prime 2}}\right)+\alpha\left[\frac{v_{r}}{\tau^{\prime}}-\left(C_{M} \Omega+F_{s}\right) u_{r}\right]
$$

where $\overline{u_{s}^{\prime 2}}, \overline{u_{s}^{\prime} v_{s}^{\prime}}, \overline{v_{s}^{\prime 2}}$ are the velocity correlations due to particle collisions and induce momentum swap in the longitudinal and radial motions of the given fraction [16].

angular momentum equation in the longitudinal direction for the particulate phase:

$$
\frac{\partial}{\partial x}\left(\alpha \omega_{s} \tilde{u}_{s}\right)+\frac{\partial}{r \partial r}\left(r \alpha \omega_{s} \tilde{v}_{s}\right)=-\frac{\partial}{\partial x}\left(\alpha \overline{u_{s}^{\prime} \omega_{s}^{\prime}}\right)-\frac{\partial}{r \partial r}\left(r \alpha \overline{v_{s}^{\prime} \omega_{s}^{\prime}}\right)-\alpha C_{\omega} \frac{\Omega}{\tau}
$$

where $\overline{u_{s}^{\prime} \omega_{s}^{\prime}}$ and $\overline{v_{s}^{\prime} \omega_{s}^{\prime}}$ are the linear-angular velocity correlations of particles due to interparticle collisions calculated according to [16].

\subsection{Boundary conditions for the RANS model}

As inlet boundary conditions, it is assumed that particles enter the previously computed, fully developed flow domain of the single-phase flow, having the initial longitudinal velocity determined by the lag coefficient. The equilibrium outlet boundary conditions were set at the exit cross-section $x=100 D$, i.e. the non-gradient derivatives from all velocities of all phases, turbulence kinetic energy and mass concentration over longitudinal coordinate were written according to [19]. Since the particulate flow in the vertical pipe is considered as axisymmetrical, the non-gradient boundary conditions were set at the pipe axis for the longitudinal velocity components of gas and particles, the turbulent energy and particle mass concentration. The boundary conditions were set zero at the pipe axis for the radial velocities of both phases and the particle angular velocity. The concept of "wall functions" [30] has been applied to set the boundary conditions at the wall. While applying the balance of the production and dissipation rate of kinetic energy "near the wall" with using the eddy-viscosity concept [31], it can link the friction velocity $v_{*}$ and shear stress $\tau_{w}$ through the turbulence kinetic energy as 
$v_{*}^{2}=\tau_{w} / \rho=c_{\mu}^{0.5} k$. The computations near the wall were carried out at the half-width of the control volume off the wall. Then, for the longitudinal velocity of the gas phase and for the turbulence energy computed by means of its production $P_{k}$, the following boundary conditions are as follows:

$$
\begin{aligned}
& \left\{\begin{array}{lc}
u=\sqrt{\frac{\tau_{w}}{\rho}} \frac{1}{x} \ln \left(y^{+}\right)+C=v_{*} \frac{1}{x} \ln \left(E \frac{y}{v} v_{*}\right) & 11.6 \leq y^{+}<500 \\
u=\frac{v_{*}^{2} y}{v} & y^{+}<11.6
\end{array},\right. \\
& P_{k}=\frac{2 \tau_{w}^{2}}{x \rho c_{\mu}^{0.25} k^{0.5} y},
\end{aligned}
$$

where empirical constant $x=0.41 ; y=\Delta / 2$ ( $\Delta$ is the width of the control volume).

The wall boundary conditions for the particulate phase have taken into account the particle's velocity lag determined through particles-wall interaction [19].

\subsection{Numerical method}

The control volume method was applied to solve mass and momentum equations of both phases by using the implicit lower and upper matrix decomposition method with fluxblending differed-correction and upwind-differencing schemes [31]. Calculations were performed in dimensional form for all flow regimes. The number of the control volumes was varied from 280000 to 1120000 , corresponding to the increase in the pipe diameter from $D=30.5$ $\mathrm{mm}$ to $D=61 \mathrm{~mm}$, and their size remained constant across the pipe flow.

\section{Numerical results}

The numerical results presented in the figures have been obtained at a distance of $x / D=100$ from the pipe entrance. At this distance it was reasonable to stipulate that the steady flow conditions have been reached and there was no influence of the entrance conditions. The results presented here are mainly dimensionless, but some of them are given in dimensional form. 250-, 500 and $750 \mu \mathrm{m}$ coal particles (physical density $\rho_{p}=1600 \mathrm{~kg} / \mathrm{m}^{3}$ ) were used in investigations. The flow mass loading was $m^{*}=1$ and $10 \mathrm{~kg}$ dust $/ \mathrm{kg}$ air. The applied particles were light enough to respond to turbulent fluctuations of gas.

The Reynolds number Re was assigned as the constant through all calculations and set equal to $4.4 \times 10^{4}$. The pipe diameter $D$ was $30.5,45.75$ and $61 \mathrm{~mm}$ for the gas average velocities $\bar{u}=21.6,14.6$ and $10.8 \mathrm{~m} / \mathrm{s}$, respectively. The average longitudinal velocity and turbulence energy radial distributions calculated for these three regimes are shown in Figures 2 and 3. 


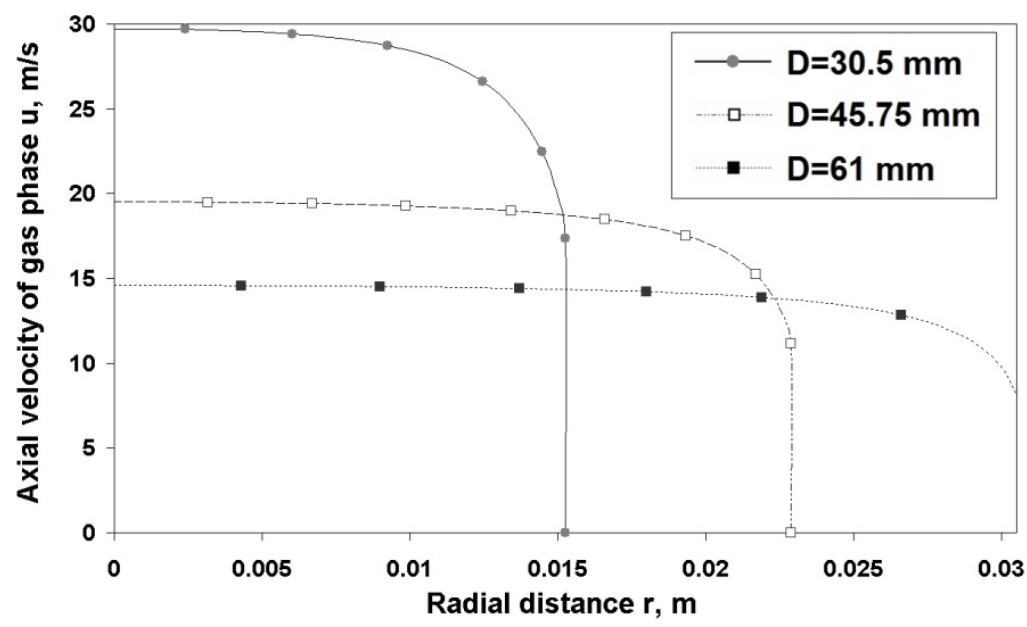

Figure 2. Profiles of the longitudinal gas velocity in the pipes $D=30.5,45.75$ and $61 \mathrm{~mm}, \operatorname{Re}=4.4 \times 10^{4}$.

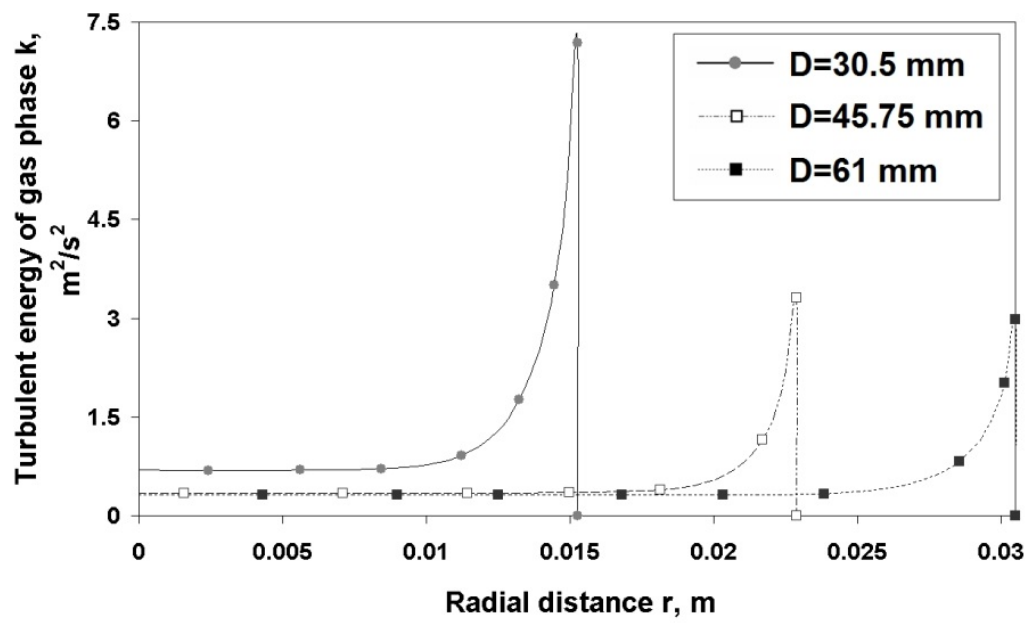

Figure 3. Profiles of the turbulence energy of gas in the pipes $D=30.5,45.75$ and $61 \mathrm{~mm}, \operatorname{Re}=4.4 \times 10^{4}$.

The profiles of particles velocity $u_{s}$ normalized to the longitudinal gas velocity, which was taken place at the pipe axis, and the particles mass concentration $\alpha$ normalized to its magnitude obtained at the pipe axis, are shown in Figure 4 for $250 \mu \mathrm{m}$ particles at the mass loading of $m^{*}=1$. The turbulence modulation TM determined as TM $=\left(k / k_{0}-1\right) \times 100 \%$, where $k$ and $k_{0}$ are the turbulence energy of the gas phase for the particulate flow conditions and the gas flow 


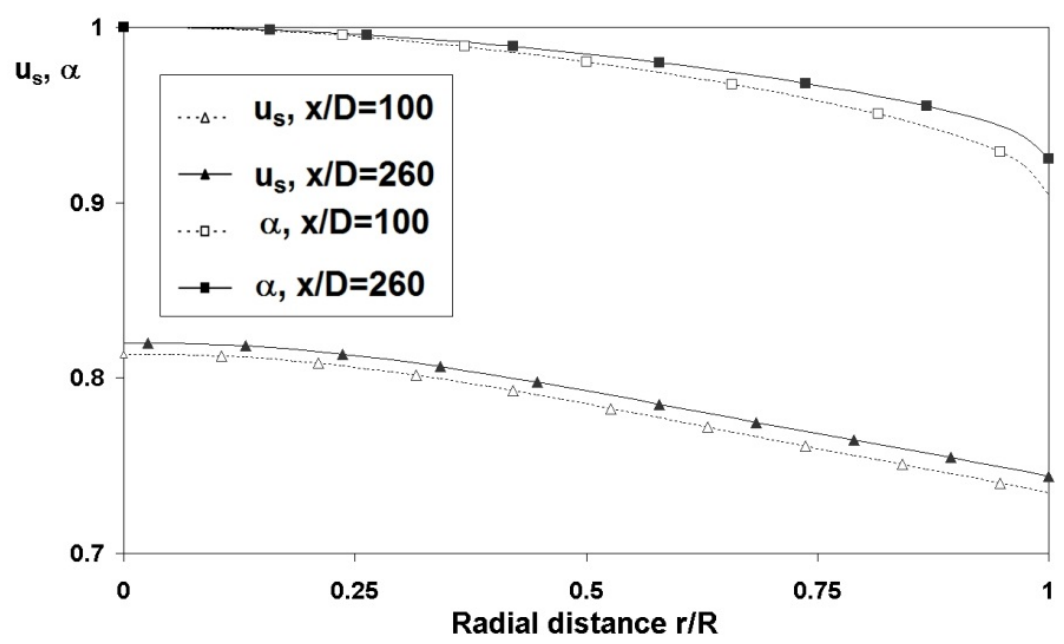

Figure 4. Profiles of the normalized longitudinal velocity of particulate phase and particle mass concentration of 250 $\mu \mathrm{m}$ coal particles in various cross-sections $x / D, m^{*}=1, D=30.5 \mathrm{~mm}, \operatorname{Re}=4.4 \times 10^{4}$.

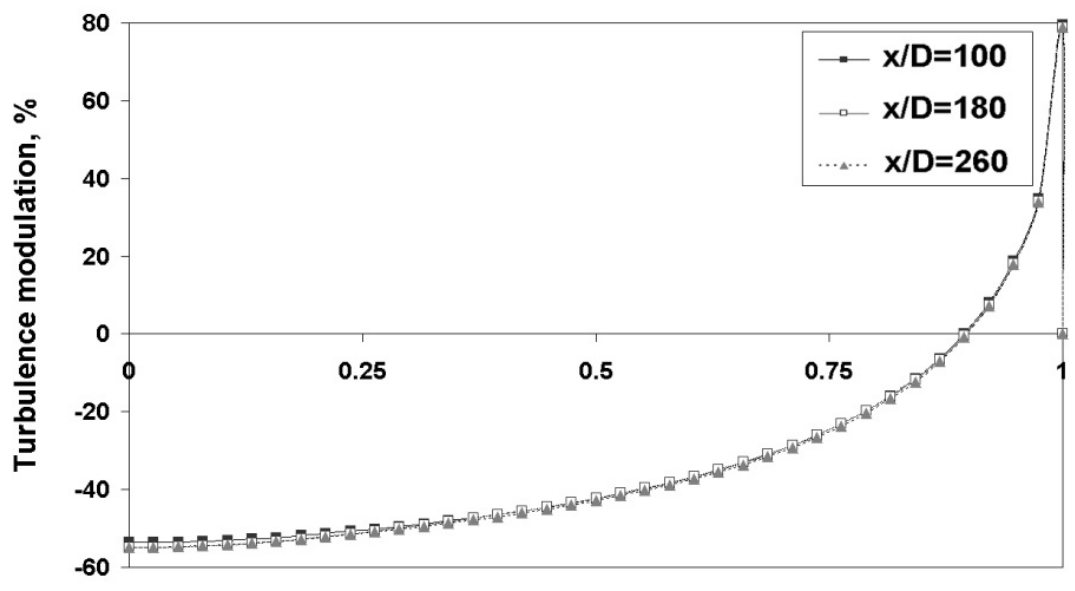

Radial distance $\mathbf{r} / \mathbf{R}$

Figure 5. Profiles of the turbulence modulation by $250 \mu \mathrm{m}$ coal particles in various cross-sections $x / D, m^{*}=1, D$ $=30.5 \mathrm{~mm}, \mathrm{Re}=4.4 \times 10^{4}$.

unladen with particles, respectively, is presented in Figure 5 for various exit cross-sections $x / D=100,180$ and 260. Based on the results shown in Figures 4 and 5, one can conclude that for the saving of computation time, the exit cross-section $x / D=100$ can be considered as the steady-state two-phase pipe flow section. 
The following figures show the influence of various force factors on cross-sectional distributions of the velocity lag, particle mass concentration and turbulence modulation originated from the particles. Separately, there were analyzed the effect of the direct (turbulence) and indirect particle-turbulence interaction (no-coupling and coupling) and the inter-particle collisions.

The analysis of behavior of the normalized longitudinal velocity lag is shown in Figure 6 for various force factors for the $250 \mu \mathrm{m}$ coal particles at $m^{*}=1$. Here and below the longitudinal velocity lag is presented as the ratio of the longitudinal velocity slip between the gas and particulate phases to the terminal velocity of particles $\left(u-u_{s}\right) / v_{t}$, where $v_{t}$ is the particle terminal velocity. One can see that larger particles have less magnitude of axial velocity lag then those of small particles with noticeable velocity difference. It looks like unexpected result, however, formation of velocity lag is multifold process.

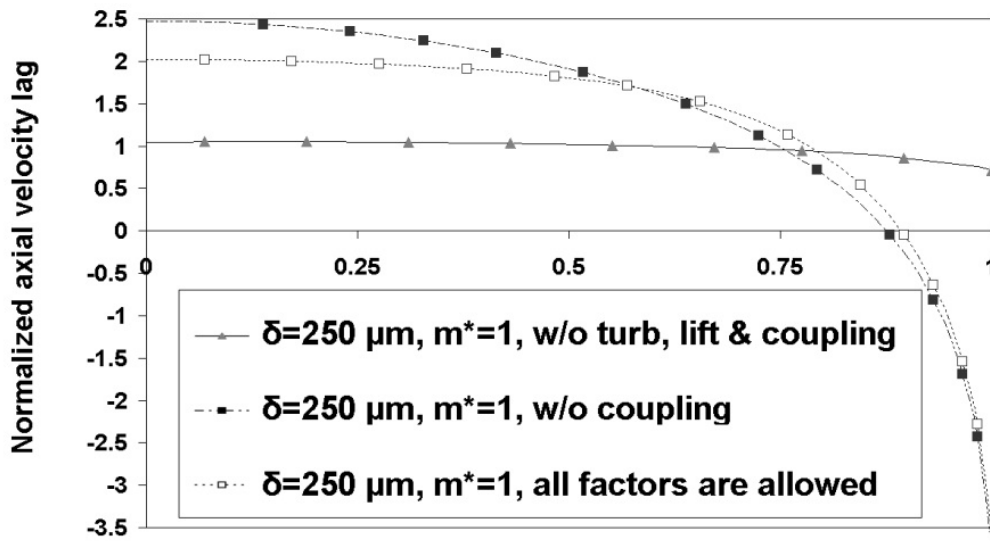

Radial distance $r / R$

Figure 6. Profiles of the normalized longitudinal velocity lag for $250 \mu \mathrm{m}$ coal particles obtained for various flow conditions, $m^{*}=1, D=45.75 \mathrm{~mm}, \operatorname{Re}=4.4 \times 10^{4}$.

If the motion of particles is exposed only by the viscous and gravitation forces (without the direct effect of turbulence, lift forces and coupling), the velocity lag between two phases approaches to the particles terminal velocity occurring in the steady-state flow domain, i.e. the ratio $u_{r} / v_{t}$ converges to unity (the curve marked by triangles, Figure 6). However, as the numerical simulation shows, if the motion of particles is exposed by various force factors, then the normalized longitudinal velocity lag increases above the particles terminal velocity.

On the face of it, the increase in the particle size should result in increase of absolute value of the velocity lag occurring for the given pipe diameter. However, the more detailed analysis shows that increase of the particles size results in reduce of the normalized longitudinal 


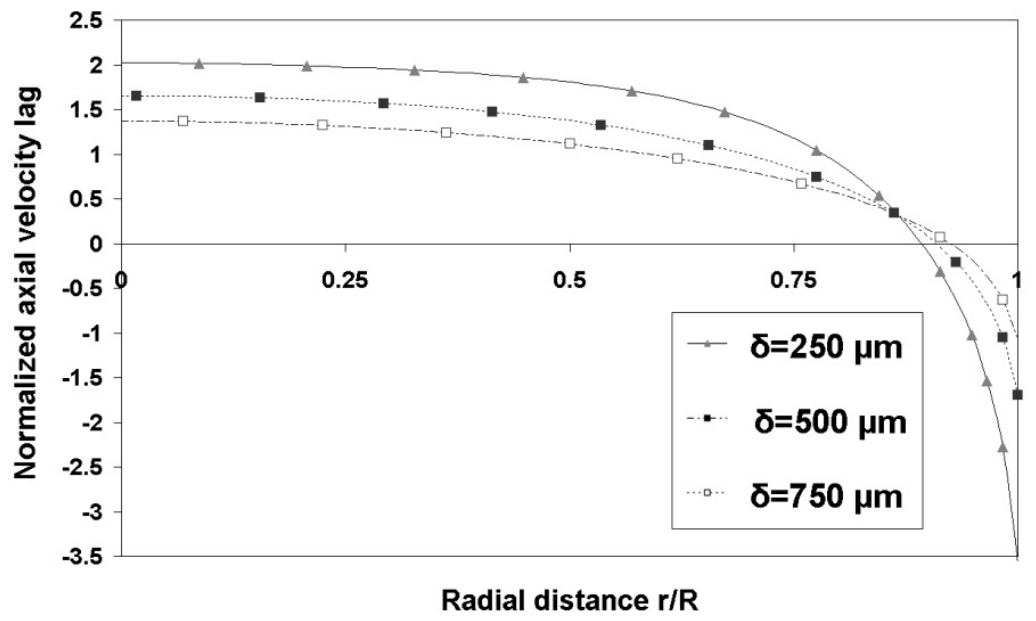

Figure 7. Profiles of the normalized longitudinal velocity lag for 250,500 and $750 \mu \mathrm{m}$ coal particles, $m^{*}=1, D=45.75$ $\mathrm{mm}, \mathrm{Re}=4.4 \times 10^{4}$.

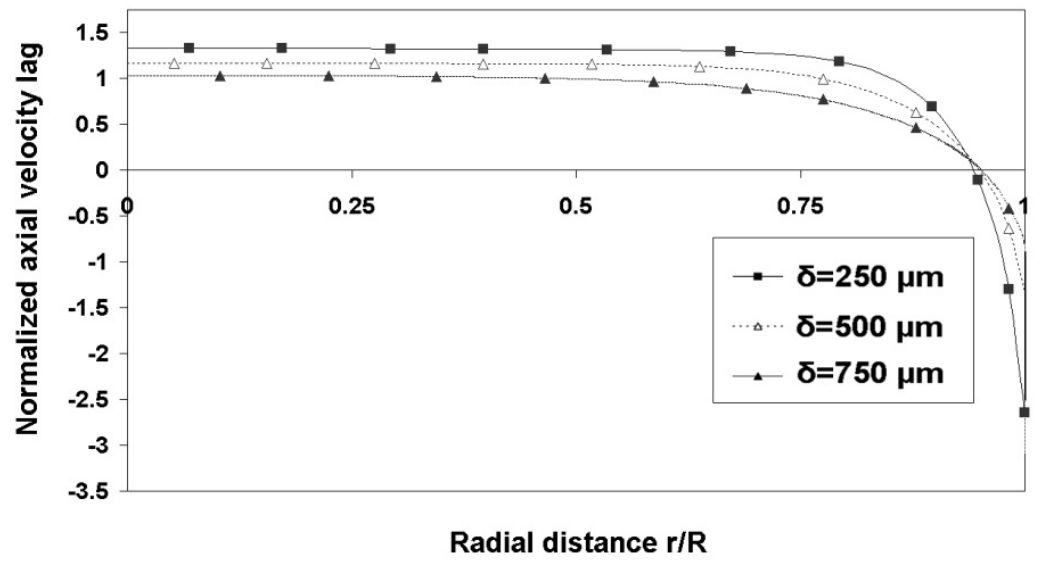

Figure 8. Effect of mass loading on longitudinal velocity lag, $m^{*}=10$; the flow conditions are the same as in Figure 7 .

velocity lag (Figures 7 and 8). This effect is more pronounced with increase of the flow mass loading (cf. Figures 7 and 8).

Diminishing of the normalized longitudinal velocity lag observed for relatively dense flow at $m^{*}=10$ (s. Figure 8 ) clearly depicts the tendency of the turbulence attenuation by particles, or, in other words, decrease of direct effect of turbulence on the particles motion. 


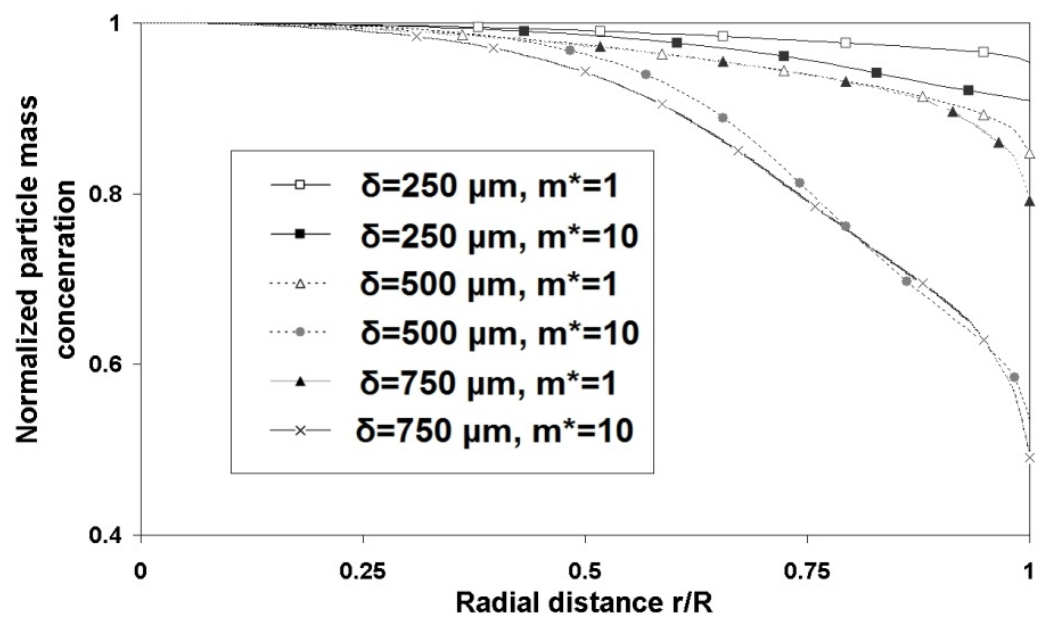

Figure 9. Profiles of the mass concentration of 250,500 and $750 \mu \mathrm{m}$ coal particles, $m^{*}=1$ and $10, D=45.75 \mathrm{~mm}$, $\operatorname{Re}=4.4 \times 10^{4}$.

In order to trace the effects of the particles size and mass loading on the turbulence modulation let us first examine the distribution of the particle mass concentration presented in Figure 9. As one can see, the growth of the particle size and flow mass loading makes profiles steeper $[16,32]$ with more pronounced tendency with respect of the particle size variation. The smaller particles are easier spread out of the pipe flow domain due to the higher value of turbulent diffusion coefficient, and the growth of the mass loading diminishes turbulence and its diffusion aligning process.

Figures 10 and 11 explicitly address to the coupling effect, which was observed for two flow mass loadings $m^{*}=1$ (Figure 10) and $m^{*}=10$ (Figure 11) for 250, 500 and $750 \mu \mathrm{m}$ coal particles. Obviously, the higher mass loading leads to the higher rate of the turbulence modulation, i.e. if there was turbulence attenuation occurred for the given particle size, then this process was intensified for the higher mass loading (cf. the corresponding curves plotted for the same particle sizes in Figures 10 and 11).

The next series of plots (Figures 12-18) show the effect of the pipe diameter for the constant Reynolds number on distribution of the normalized velocity lag, the particle mass concentration and the turbulence modulation.

Figures 12, 13 and 14 show the profiles of the normalized longitudinal velocity lag obtained for various pipe diameters for 250,500 and $750 \mu \mathrm{m}$ coal particles. One can see that decrease of the pipe diameter results in the higher velocity lag and, as a result, in the stronger particles involvement into the turbulent motion. This fact is proved by the data of Figures 2 and 3 showing that the smaller pipe diameter corresponds to the higher level of the turbulence energy, and, sequentially to the higher rate of the particles involvement by the gas flow. 


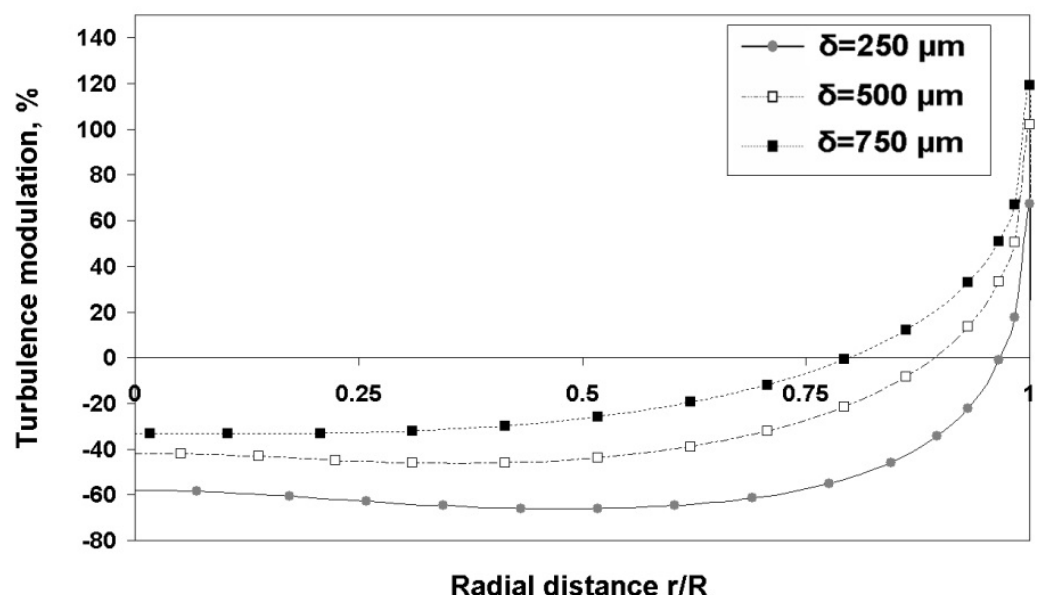

Figure 10. Profiles of the turbulence modulation by 250,500 and $750 \mu \mathrm{m}$ coal particles, $m^{*}=1, D=45.75 \mathrm{~mm}$, $\operatorname{Re}=4.4 \times 10^{4}$.

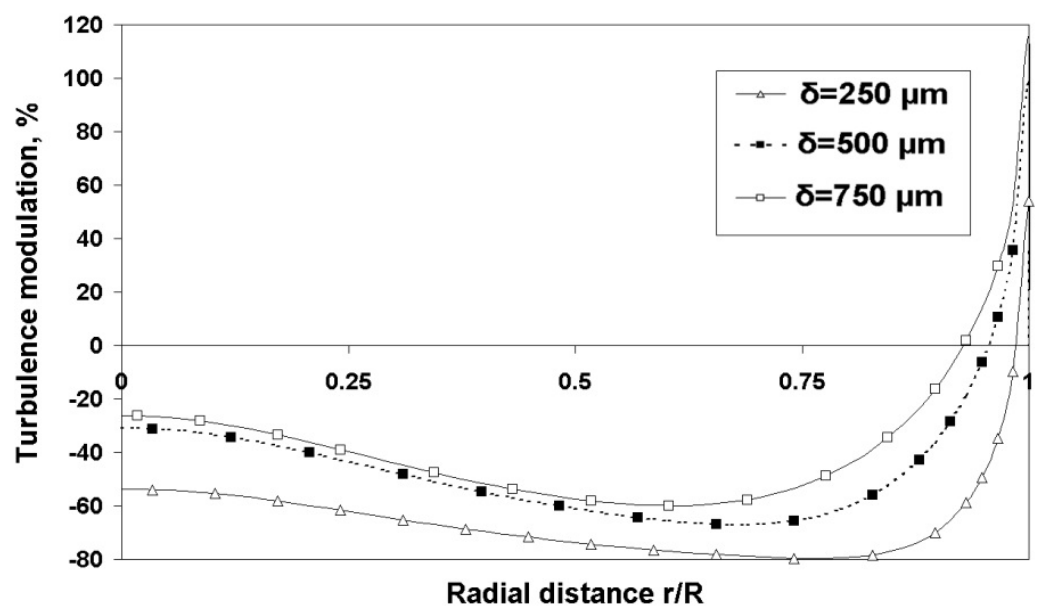

Figure 11. Effect of the mass loading on the turbulence modulation by 250,500 and $750 \mu \mathrm{m}$ coal particles, $m^{*}=10$, $D=45.75 \mathrm{~mm}, \mathrm{Re}=4.4 \times 10^{4}$. 


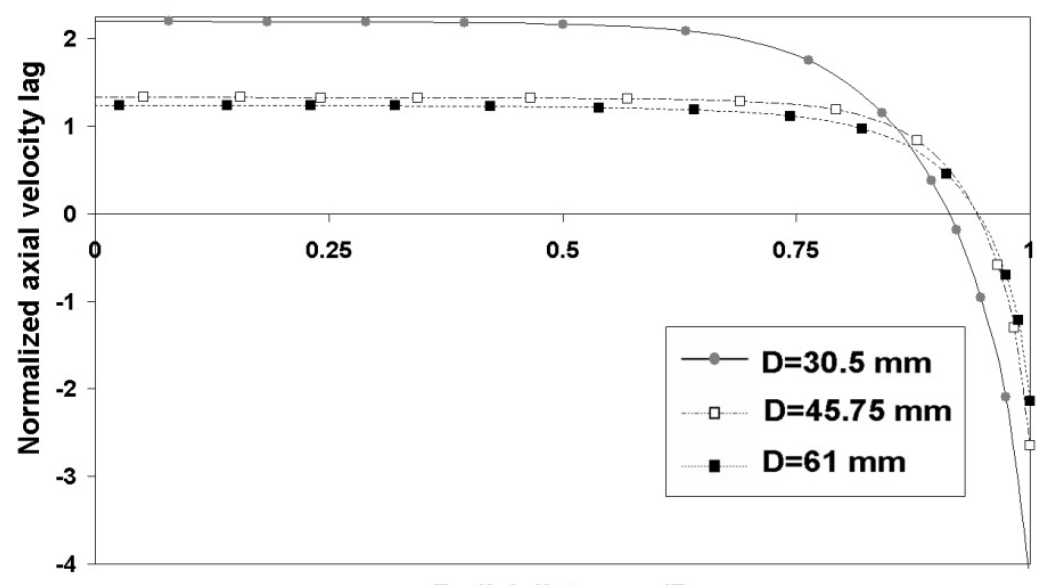

Radial distance $r / R$

Figure 12. Profiles of the normalized longitudinal velocity lag for $250 \mu \mathrm{m}$ coal particles in the pipes $D=30.5,45.75$ and $61 \mathrm{~mm}, m^{*}=10, \operatorname{Re}=4.4 \times 10^{4}$.

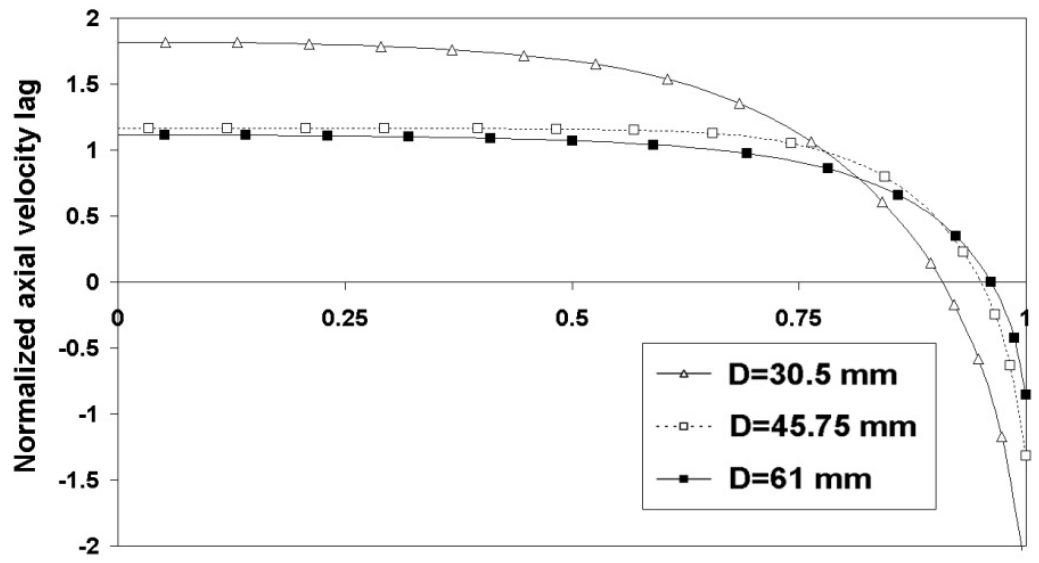

Radial distance $r / R$

Figure 13. Profiles of the normalized longitudinal velocity lag for $500 \mu \mathrm{m}$ coal particles in the pipes $D=30.5,45.75$ and $61 \mathrm{~mm}, m^{*}=10, \operatorname{Re}=4.4 \times 10^{4}$. 


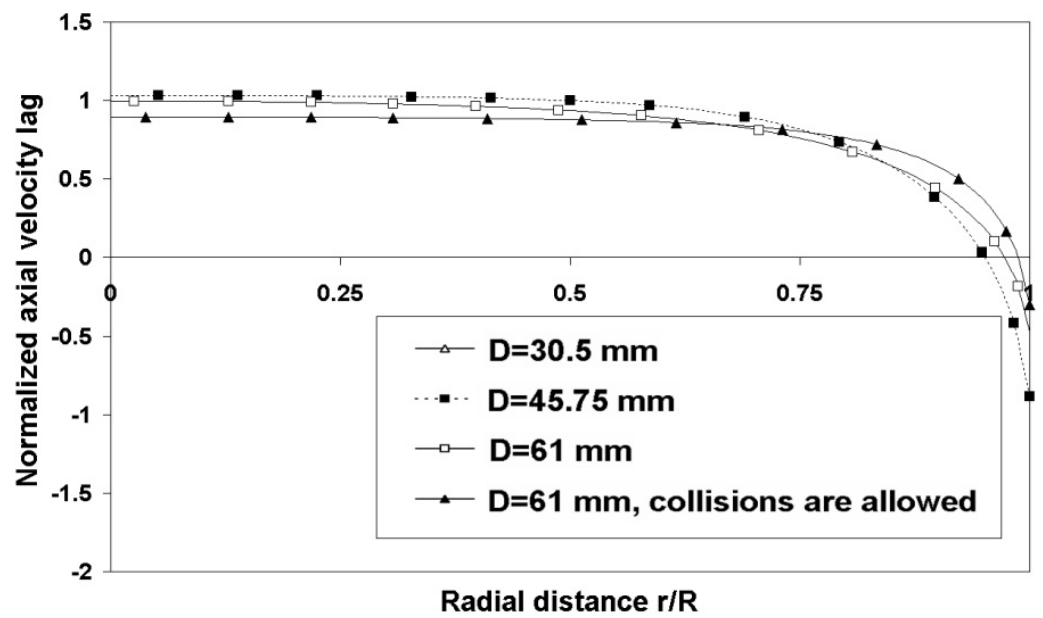

Figure 14. Profiles of the normalized longitudinal velocity lag for $750 \mu \mathrm{m}$ coal particles in the pipes $D=30.5,45.75$ and $61 \mathrm{~mm}, m^{*}=10, \operatorname{Re}=4.4 \times 10^{4}$.

The effect of the particles collisions that may occur at the higher mass loading of $m^{*}=10$ (s. Figure 14) brings this process to slow down the particles motion. Therefore, the particles collisions result in the decrease of the normalized velocity slip as compared with the case of no collisions (cf. Figures 12, 13 and 14).

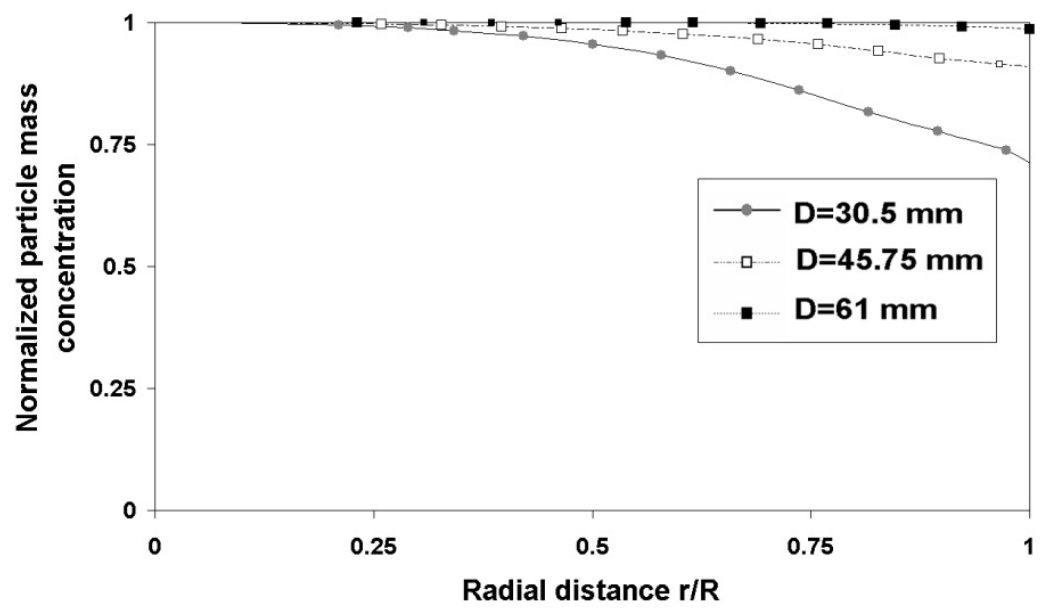

Figure 15. Profiles of the normalized mass concentration for $250 \mu \mathrm{m}$ coal particles in the pipes $D=30.5,45.75$ and 61 $\mathrm{mm}, \mathrm{m}^{*}=10, \operatorname{Re}=4.4 \times 10^{4}$. 
One can see that the effect of the pipe diameter has the same tendency as the effect of the particle size, i.e. the increase of the pipe diameter acts like the decrease of the particle size, straightening the profiles of the particle mass concentration (Figures 15 and 16). An accounting of the inter-particle collision effect intensifies the particle dispersion (s. Figure 16).

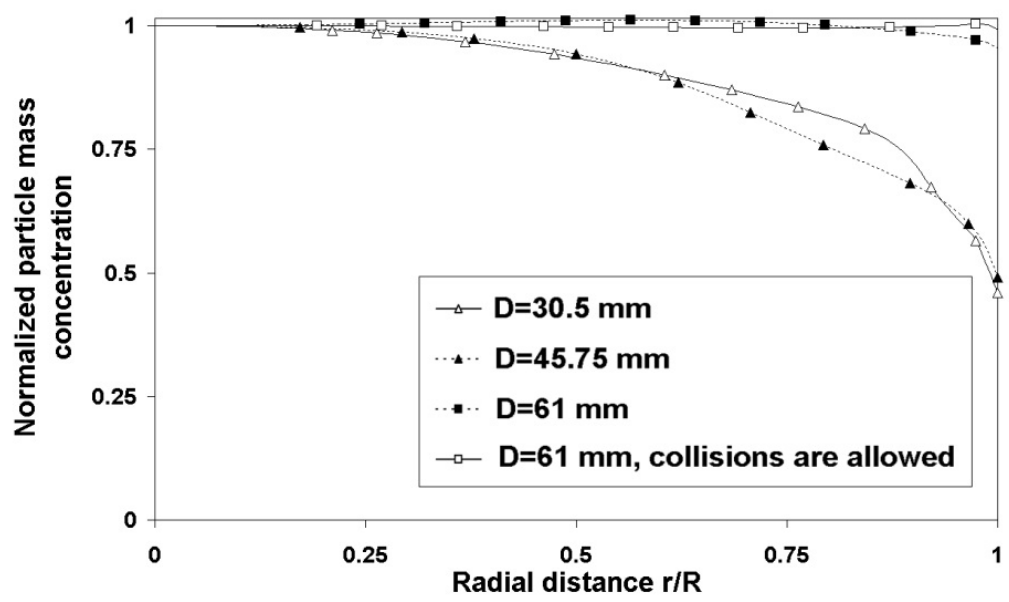

Figure 16. Profile of the normalized mass concentration for $750 \mu \mathrm{m}$ coal particles in the pipes $D=30.5,45.75$ and 61 $m m, m^{*}=10, \operatorname{Re}=4.4 \times 10^{4}$.

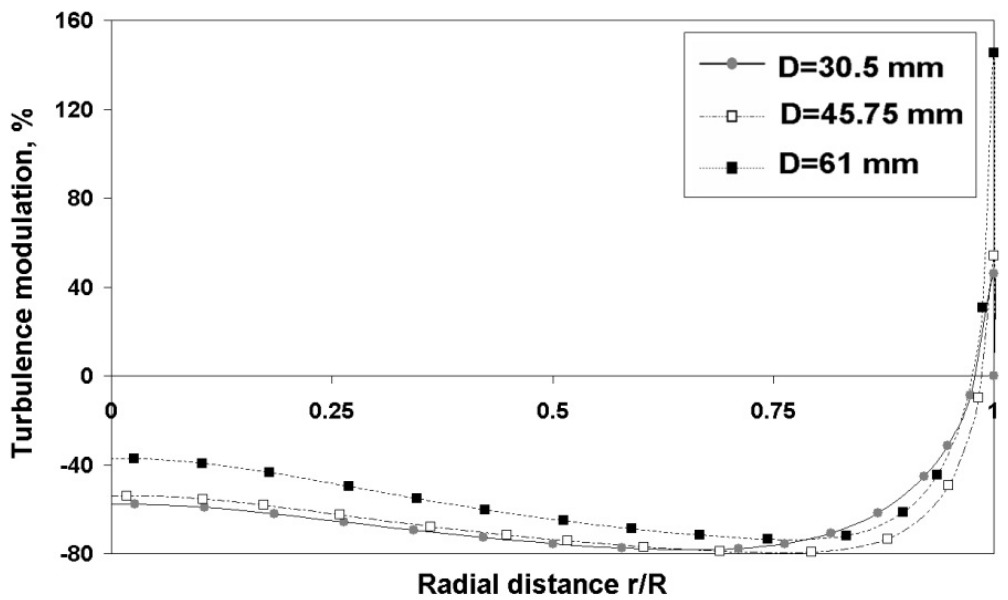

Figure 17. Profiles of the turbulence modulation for $250 \mu \mathrm{m}$ coal particles in the pipes $D=30.5,45.75$ and $61 \mathrm{~mm}$, $m^{*}=10, \operatorname{Re}=4.4 \times 10^{4}$. 


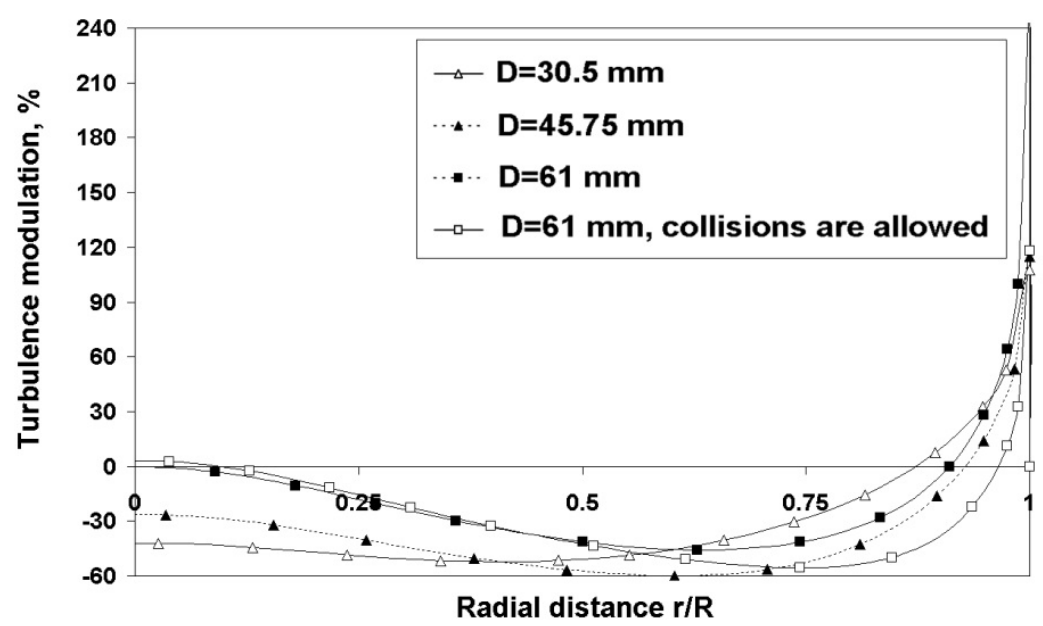

Figure 18. Profiles of the turbulence modulation for $750 \mu \mathrm{m}$ coal particles in the pipes $D=30.5,45.75$ and $61 \mathrm{~mm}$, $m^{*}=10, \operatorname{Re}=4.4 \times 10^{4}$.

The turbulence modulation is shown in Figures 17 and 18 for the considered particles sizes in two marginal cases: $\delta=250$ and $750 \mu \mathrm{m}$. It is evident that the increase of the particle size leads to decrease of the attenuation rate of turbulence. The effect of the inter-particle collisions (Figure 18) results in the enhancement of turbulence by particles in vicinity of the flow axis and its damping, that occurs in the region locating between the flow axis and the pipe wall.

\section{Conclusions}

2D RANS numerical method fitted with the appropriate closure equations was applied for the computational investigation of the upward turbulent particulate pipe flow at the distance of 100 calibers from the pipe entrance. The axial velocity lag, turbulent kinetic energy of gas and particles mass concentration, effected by the gravity, viscous drag, the particle-turbulence, particle-particle, particle-wall interactions as well as the Saffman and Magnus lift forces, were examined for various particle sizes and flow mass loadings at the same flow Reynolds number.

The obtained numerical results allow to draw the following conclusions pertaining to behavior of solid particles under the conditions of the upward turbulent pipe flow:

1. It is obvious that if the motion of particles is exposed only by the viscous and gravitational forces (without the direct effect of turbulence, lift forces and coupling), the absolute magnitude of the axial velocity lag approaches to the particles terminal velocity. However, simultaneous accounting of all force factors, effecting on the fine particles, results in substantial exceeding of their axial velocity lag as compared with their terminal velocity, that is due to intensification of influence of turbulence on a motion of the fine particles. 
2. It was revealed that the effect of the particles size appears as follows:

- the increase of the particles size results in reducing of the relative axial velocity lag. The absolute magnitude of the velocity lag approaches to the particles terminal velocity;

- the fine particles spread more uniformly in the cross-section of the pipe as against the coarse ones, due to their higher coefficient of turbulent diffusion;

- enlargement of the particles size gives the lower rate of the turbulence attenuation.

3. The given investigation shows that the effect of the flow mass loading acts in the following way:

- the increase of the flow mass loading causes the diminution of the relative velocity lag, and this is more pronounced for the fine particles. The same tendency also takes place when considering the inter-particle collisions for the large flow loading;

- the increase of the flow loading results in the turbulence attenuation that is followed by the non-uniform cross-sectional distributions of the particles mass concentration, while the accounting of the inter-particle collisions causes the opposite trend, i.e. their flattening.

4. The effect of the pipe diameter acts in the way that its increase: a) gives rise to decrease of the relative velocity lag, b) results in flattening of the cross-sectional distributions of the particles mass concentration and c) induces the decrease of the turbulence attenuation rate.

\section{Acknowledgements}

The work was done within the frame of the target financing under the Project SF0140070s08 (Estonia) and supported by the ETF grant Project ETF9343 (Estonia). The authors are grateful for the technical support of Computational Biology Initiative High Performance Computing Center of University of Texas at San Antonio (USA) and Texas Advanced Computing Center in Austin (USA). This study is related to the activity of the European network action COST MP1106 "Smart and green interfaces - from single bubbles and drops to industrial, environmental and biomedical applications".

\section{Author details}

Alexander Kartushinsky*, Ylo Rudi, Igor Shcheglov, Sergei Tisler and Igor Krupenski

*Address all correspondence to: aleksander.kartusinski@ttu.ee

Research Laboratory of Multiphase Media Physics, Faculty of Science, Tallinn University of Technology, Tallinn, Estonia 


\section{References}

[1] Pfeffer R., Rosetti S., Licklein S. Analysis and Correlation of Heat Transfer Coefficient and Heat Transfer Data for Dilute Gas-Solid Suspensions. NASA Rep. TND-3603, 1966.

[2] Davies J.T. Calculation of critical velocities to maintain solids in suspension in horizontal pipes. Chemical Engineering Science 1987; 42(7) 1667-1670.

[3] Gore R.A., Crowe C.T. Effect of Particle Size on Modulating Turbulent Intensity. International Journal of Multiphase Flow 1989; 15(2) 279-285.

[4] Tsuji, Y. Morikawa, Y. LDV Measurements of an Air-Solid Two-Phase Flow in a Horizontal Pipe. Journal of Fluid Mechanics 1982; 120 385-409.

[5] Michaelides E.E. A Model for the Flow of Solid Particles in Gases. International Journal of Multiphase Flow 1984; 10(1) 61-77.

[6] Tsuji Y., Morikawa Y., Shiomi H. LDV Measurements of an Air-Solid Two-Phase Flow in a Vertical Pipe. Journal of Fluid Mechanics 1984;139 417-434.

[7] Squires K.D., Eaton J. K. Particle Response and Turbulence Modification in Isotropic Turbulence. Physics of Fluids 1990; 2 1191-1203.

[8] Yuan Z., Michaelides E.E. Turbulence Modulation in Particulate Flows - a Theoretical Approach. International Journal of Multiphase Flow 1992; 18(5) 779-785.

[9] Gidaspow D. Multiphase Flow and Fluidization: Continuum and Kinetic Theory Descriptions. Boston: Acad. Press; 1994.

[10] Cabrejos F.J, Klinzing G.E. Pickup and Saltation Mechanisms of Solid Particles in Horizontal Pneumatic Transport. Powder Technology 1994; 79(2) 173-186.

[11] Yarin L.P., Hetsroni G. Turbulence Intensity in Dilute Two-Phase Flows. Parts I, II and III International Journal of Multiphase Flow 1994; 20(1) 1-15.

[12] Cao J, Ahmadi G. Gas-Particle Two-Phase Turbulent Flow in Vertical Duct. International Journal of Multiphase Flow 1995; 21(6) 1203-1228.

[13] Crowe C.T., Gillandt I. Turbulence modulation of fluid-particle flows - a basic approach. In: Proceedings of the 3rd International Conference on Multiphase Flow, 8-12 June 1998, Lyon, France. CD-ROM.

[14] Crowe C. T. On Models for Turbulence Modulation in Fluid-Particle Flows. International Journal of Multiphase Flow 2000; 26(5) 719-727.

[15] Sommerfeld M. Analysis of Collision Effects for Turbulent Gas-Particle Flow in a Horizontal Channel. Part I: Particle Transport. International Journal of Multiphase Flow 2003; 29(4) 675-699. 
[16] Kartushinsky A., Michaelides E.E. An analytical approach for the closure equations of gas-solid flows with inter-particle collisions. International Journal of Multiphase Flow 2004; 30(2) 159-180.

[17] Michaelides EE. Particles, Bubbles and Drops - Their Motion, Heat and Mass Transfer. New Jersey: World Scientific Publishers; 2006.

[18] Kartushinsky A.I., Michaelides E.E., Zaichik L.I. Comparison of the RANS and PDF Methods for Air-Particle Flows. International Journal of Multiphase Flow 2009; 35(10) 914-923.

[19] Kartushinsky A.I., Michaelides E.E., Hussainov M., Rudi Y. Effects of the Variation of Mass Loading and Particle Density in Gas-Solid Particle Flow in Pipes. Powder Technology 2009; 193(2) 176-181.

[20] Kartushinsky A.I., Michaelides E.E., Rudi Y.A., Tisler S.V., Shcheglov I.N. Numerical Simulation of Three-Dimensional Gas-Solid Particle Flow in a Horizontal Pipe. American Institute of Chemical Engineers 2011; 57(11) 2977-2988.

[21] Elghobashi S.E., Abou-Arab T.W. A Two-Equation Turbulence Model for Two-Phase Flows. Physics of Fluids 1983; 26(4) 931-938.

[22] Rizk M.A., Elghobashi S.E. A Two-Equation Turbulence Model for Dispersed Dilute Confined Two-Phase Flows. International Journal of Multiphase Flow 1989; 15(1) 119-133.

[23] Simonin O. Eulerian formulation for particle dispersion in turbulent two-phase flows. In: Sommerfeld M, Wennerberg D. (eds.) Proceedings of the 5th Workshop on Two-Phase Flow Predictions, 19-22 March 1990, Erlangen, Germany. Ju®lich: Forschungszentrum Ju®lich; 1990.

[24] Deutsch E., Simonin O. Large eddy simulation applied to the motion of particles in stationary homogeneous fluid turbulence. In: Michaelides EE, Fukano T, Serizawa A. (eds.) Proceedings of the 1st ASME/JSME Fluids Engineering Conference, 23-27 June 1991, Portland, USA. New York: American Society of Mechanical Engineers, Series FED; 1991.

[25] Reeks M.W. On the Continuum Equations for Dispersed Particles in Nonuniform Flows. Physics of Fluids A: Fluid Dynamics 1992; 4(6) 1290-1303.

[26] Marchioli C., Giusti A., Salvetti M.-V., Soldati A. Direct Numerical Simulation of Particle Wall Transfer and Deposition in Upward Turbulent Pipe Flow. International Journal of Multiphase Flow 2003; 29(6) 1017-1038.

[27] Schiller L., Naumann A. Über die grundlegenden Berechnungen bei der Schwerkraftaufbereitung. Zeitschrift des Vereines Deutscher Ingenieure 1933; 77(12) 318-320. 
[28] Mei R. An Approximate Expression for the Shear Lift Force on a Spherical Particle at Finite Reynolds Number International Journal of Multiphase Flow 1992; 18(1) 145-147.

[29] Zaichik L.I., Alipchenkov V.M. Statistical Models for Predicting Particle Dispersion and Preferential Concentration in Turbulent Flows. International Journal of Heat and Fluid Flow 2005; 26(3) 416-430.

[30] Pope SB. Turbulent Flows. Cambridge - New York: Cambridge University Press; 2008.

[31] Perić M., Scheuerer G. CAST - A Finite Volume Method for Predicting Two-Dimensional Flow and Heat Transfer Phenomena. GRS - Technische Notiz SRR-89-01. 1989.

[32] Kartushinsky A., Michaelides E. E. Particle-Laden Gas Flow in Horizontal Channels with Collision Effects. Powder Technology 2006; 168(2) 89-103. 